\title{
Fatores associados à ideação suicida em cuidadores de sobreviventes de acidente vascular encefálico
}

\author{
Factors associated with suicidal ideation in caregivers of stroke survivors
}

\author{
Como citar este artigo: \\ Costa TF, Bezerra TA, Pimenta CJL, Silva CRR, Ferreira GRS, Costa KNFM. Factors associated with suicidal ideation in caregivers of stroke \\ survivors. Rev Rene. 2020;21:e42171. DOI: https://doi.org/10.15253/2175-6783.20202142171
}

(D)Tatiana Ferreira da Costa ${ }^{1}$
(D) Thaíse Alves Bezerra ${ }^{1}$
(D) Cláudia Jeane Lopes Pimenta ${ }^{1}$
(D) Cleane Rosa Ribeiro da Silva ${ }^{1}$
(D) Gerlania Rodrigues Salviano Ferreira ${ }^{1}$
(D) Kátia Neyla de Freitas Macedo Costa ${ }^{1}$

${ }^{1}$ Universidade Federal da Paraíba. João Pessoa, PB, Brasil.

\section{Autor correspondente:}

Cláudia Jeane Lopes Pimenta

Rua Luiz Prímola da Silva, 30, Bancários. CEP: 58051-340. João Pessoa, PB, Brasil.

E-mail: claudinhajeane8@hotmail.com

\begin{abstract}
RESUMO
Objetivo: identificar os fatores associados à presença de ideação suicida em cuidadores de sobreviventes de acidente vascular encefálico. Métodos: pesquisa transversal, realizada com 151 cuidadores informais primários. Utilizaram-se da Escala de Depressão, Ansiedade e Estresse-21 (Depression Anxiety and Stress Scale) e de questionário para avaliação da presença de pensamentos de ideação suicida, cujos dados foram analisados por estatística descritiva e inferencial. Resultados: a maior parte dos cuidadores apresentou níveis normais de depressão $(76,8 \%)$, ansiedade $(76,2 \%)$ e estresse $(79,5 \%)$, assim como a ausência de pensamentos de ideação suicida $(70,9 \%)$. A correlação entre as escalas evidenciou que à medida que os níveis de ansiedade, depressão e estresse aumentam, os pensamentos de ideação suicida se elevam de maneira proporcional. Conclusão: observou-se que níveis elevados de depressão, ansiedade e estresse favoreceram o aumento da frequência de pensamentos de ideação suicida entre os cuidadores de sobreviventes de acidente vascular encefálico.
\end{abstract}

Descritores: Cuidadores; Ansiedade; Depressão; Estresse Psicológico; Ideação Suicida.

\section{ABSTRACT}

Objective: to identify factors associated with the presence of suicidal ideation in caregivers of stroke survivors. Methods: cross-sectional survey conducted with 151 primary informal caregivers. The Depression Anxiety and Stress Scale-21 and a questionnaire were used to evaluate the presence of thoughts of suicidal ideation, whose data were analyzed by descriptive and inferential statistics. Results: most caregivers had normal levels of depression (76.8\%), anxiety $(76.2 \%)$ and stress $(79.5 \%)$, and showed no thoughts of suicidal ideation $(70.9 \%)$. The correlation between scales showed that thoughts of suicidal ideation rise proportionally to the increase of anxiety, depression and stress levels. Conclusion: it was observed that high levels of depression, anxiety and stress favored the increased frequency of suicidal thoughts among caregivers of stroke survivors.

Descriptors: Caregivers; Anxiety; Depression; Stress, Psychological; Suicidal Ideation. 


\section{Introdução}

As doenças crônicas não transmissíveis são as principais causas de morbidade e mortalidade no mundo, gerando impactos que ultrapassam a esfera individual e afetam famílias, comunidade e sociedade. Dentre estas, o acidente vascular encefálico é o distúrbio cerebrovascular mais comum, representando uma das principais causas de morte e incapacidades em todo o mundo ${ }^{(1)}$.

0 acidente vascular encefálico é considerado síndrome de início súbito, causado pela interrupção do transporte de sangue para o cérebro, em que as manifestações clínicas compreendem ampla variedade de déficits motores, auditivos, visuais, intelectuais e neurológicos que podem ser temporários ou permanentes ${ }^{(2)}$. Devido aos avanços científicos na área médica, vivenciados nas últimas décadas, tem-se observado número crescente de pessoas que sobrevivem ao acidente vascular encefálico, contudo, em virtude do grau de comprometimento das funções corporais provocado pelo episódio, muitos necessitam de assistência hospitalar de alta complexidade e de cuidados prolongados na residência, surgindo a figura do cuidador $^{(3)}$.

Existem dois tipos de cuidadores: os formais, aqueles que possuem algum tipo de treinamento e/ ou capacitação e são pagos para realizar o serviço; e os informais, cujo cuidado é prestado, geralmente, por algum familiar próximo, principalmente cônjuge e filhos, os quais não apresentam conhecimentos para prestar esse cuidado ${ }^{(4)}$. Em decorrência da natureza repentina do acidente vascular encefálico, a família se encontra despreparada para lidar com as consequências desse evento, o que causa grande impacto para o paciente e o cuidador, o qual assume novo papel e precisa se adaptar a essa nova rotina ${ }^{(5)}$.

A experiência de cuidar de um indivíduo dependente é referida na literatura como uma tarefa desgastante e que gera prejuízos para saúde e qualidade de vida do cuidador, uma vez que muitos abdicam das atividades de rotina em detrimento do cuidado ao fami- liar sobrevivente de acidente vascular encefálico ${ }^{(1-3)}$. Diante disso, os cuidadores estão frequentemente expostos a altas cargas de estresse, tornando-se vulneráveis ao adoecimento, sobretudo, ao desenvolvimento de problemas psíquicos ${ }^{(5-6)}$.

Dados semelhantes foram evidenciados com 115 cuidadores de sobreviventes de acidente vascular encefálico na Nigéria, em que se constatou alta prevalência de depressão entre os participantes, correspondendo a $46,0 \%{ }^{(7)}$. No Peru, os cuidadores afirmaram que vivenciavam constantemente situações de sofrimento como resultado do cuidado ao familiar dependente, apresentando, principalmente, sintomas depressivos, como cansaço, tristeza, mudanças de humor, insônia, ansiedade, desmotivação, desespero, angústia e ideação suicida ${ }^{(4)}$.

Nesse sentido, embora o cuidado a pacientes com sequelas de acidente vascular encefálico seja algo imprescindível para reabilitação e melhoria do quadro de saúde destes, essa atividade traz repercussões negativas para o cuidador, sendo necessário o desenvolvimento de estratégias para prevenir a sobrecarga, a depressão e o suicídio, reduzir o estresse e proporcionar melhor qualidade de vida ${ }^{(8)}$.

Assim, objetivou-se identificar os fatores associados à presença de ideação suicida em cuidadores de sobreviventes de acidente vascular encefálico.

\section{Métodos}

Trata-se de estudo transversal, realizado com cuidadores informais primários de pessoas com sequela de acidente vascular encefálico, cadastrados nas Unidades de Saúde da Família, do município de João Pessoa, Paraíba, Brasil. Definiram-se como critérios de inclusão: ter idade igual ou superior a 18 anos e ser cuidador informal primário de pessoas com sequela de acidente vascular encefálico, cadastrados nas Unidades de Saúde da Família do município. Excluíram-se os indivíduos que prestavam cuidados por período inferior a seis meses.

A seleção das Unidades de Saúde da Família foi 
realizada de forma aleatória, sendo sorteadas três unidades de cada distrito sanitário. A rede de atenção à saúde desse munícipio é composta por 194 Unidades de Saúde da Família, administradas de forma organizacional, por meio de cinco Distritos Sanitários.

0 cálculo da amostra foi baseado no quantitativo de pessoas acometidas por acidente vascular encefálico que eram cadastradas nas Unidades de Saúde da Família de João Pessoa, durante o ano de 2016, totalizando 249 indivíduos. 0 tamanho da amostra foi definido utilizando-se do cálculo para populações finitas com proporções conhecidas, tendo-se como base margem de erro de $5 \%$ (erro=0,05), com grau de confiabilidade de $95 \%\left(\alpha=0,05\right.$, que fornece $\left.\mathrm{Z}_{0,05 / 2}=1,96\right)$, considerando a proporção verdadeira como de $50 \%$ $(\mathrm{p}=0,50)$ para presença de sequelas, resultando em amostra de 151 cuidadores, os quais foram selecionados de forma proporcional ao quantitativo de indivíduos em cada unidade e distrito.

Os dados foram coletados entre setembro e dezembro de 2017, por pesquisadores previamente treinados, em processo que envolveu a apresentação, a explicação e a aplicação dos instrumentos entre os entrevistadores para padronizar a coleta de dados. Realizaram-se entrevistas individuais nos domicílios, com duração média de 30 minutos, sendo o conteúdo das falas gravado em áudio.

A identificação dos cuidadores ocorreu mediante o contato com os enfermeiros de cada unidade, solicitando informações a respeito das pessoas que sofreram acidente vascular encefálico e apresentavam algum tipo de sequela. Utilizou-se de instrumento para obtenção de dados sociodemográficos e aspectos relacionados ao cuidado prestado, contendo as variáveis sexo, idade, conjugalidade, escolaridade, renda familiar, grau de parentesco, idade do sobrevivente, reside no mesmo local que o alvo do cuidado, quantidade de dias por semana e de horas diárias destinadas para essa atividade, período de tempo que cuidado do paciente, cuidados prestados e sequelas apresentadas pelo paciente. Além disso, foram empregadas a Escala de Depressão, Ansiedade e Estresse-21 (Depression
Anxiety and Stress Scale $)^{(9)}$ e questionário para avaliação da presença de pensamentos de ideação suicida.

A escala é composta por 21 itens, avaliados em escala do tipo Likert de quatro pontos, variando de 0 (discordo totalmente) a 3 (concordo totalmente). É dividida em três subescalas, em que cada uma é formada por sete questões, destinadas a avaliar os estados emocionais de ansiedade, depressão e estresse durante a última semana. A subescala de ansiedade avalia a excitação do sistema nervoso autônomo; efeitos musculoesqueléticos; ansiedade situacional; experiências subjetivas de ansiedade. A subescala de depressão avalia sintomas como inércia; anedonia; disforia; falta de interesse e envolvimento; autodepreciação; desvalorização da vida e desânimo. E a subescala de estresse analisa a dificuldade em relaxar; excitação nervosa; fácil perturbação/agitação; irritabilidade/reação exagerada e impaciência ${ }^{(9)}$.

O cálculo do resultado individual de depressão, ansiedade e estresse corresponde ao somatório das pontuações dos itens em cada subescala. A classificação dos escores é realizada da seguinte forma: normal (depressão 0-9; ansiedade 0-7; estresse 0-14); leve (depressão 10-13; ansiedade 8-9; estresse 15-18); moderado (depressão 14-20; ansiedade 10-14; estresse 19-25); severo (depressão 21-27; ansiedade 15-19; estresse 26-33); muito severo (depressão $\geq 28$; ansiedade $\geq 20$; estresse $\geq 34)^{(9)}$.

A ideação suicida foi avaliada por quatro perguntas direcionadas a investigar quantos dias, na última semana, o sujeito apresentou os seguintes pensamentos: não consegui seguir em frente, queria desistir de tudo; tenho pensamentos sobre a morte; sentia que minha família estaria melhor se eu estivesse morto(a); pensei em me matar. A classificação do instrumento foi realizada de acordo com o total de pensamentos de ideação suicida apresentados na última semana, variando de 0 a 28, em que 0 corresponde a nenhum, 4 - 8 ( 1 a 2 vezes/semana), 12 - 16 (3 a 4 vezes/semana) e 20 - 28 ( 5 ou mais vezes/semana). Foi definida como a presença de ideação suicida quando o indivíduo exibia escore igual ou superior a 12 . 
O questionário foi elaborado a partir de busca na literatura nacional e internacional e passou por validação de face para possíveis correções e adaptações. A validação de face/rosto é um subtipo da validação de conteúdo, utilizada para verificar a adequabilidade do instrumento em medir o que se propõ $\mathrm{e}^{(10)}$. Neste sentido, a validação foi realizada por três professoras doutoras em Enfermagem, as quais fizeram sugestões para melhoria do instrumento. Após essa etapa, realizou-se teste-piloto com 20 pacientes para analisar a existência de incongruências na compreensão dos questionamentos.

Os dados foram armazenados em planilha eletrônica, estruturada no Programa Microsoft Excel, com dupla digitação, visando garantir a confiabilidade na compilação dos dados. Em seguida, foram organizados, codificados, importados e processados pelo aplicativo Statistical Package for the Social Science for Windows, versão 22.0, sendo analisados por meio de estatística descritiva e inferencial. Para verificação da normalidade/simetria dos dados numéricos, adotou-se o teste de Kolmogorov-Smirnov. A correlação entre as escalas foi realizada por meio do Coeficiente Correlação de Spearman. 0 nível de significância utilizado para as análises estatísticas foi $5 \%(\mathrm{p} \leq 0,05)$.

0 estudo foi desenvolvido de acordo com o preconizado pela Resolução no 466/2012, do Conselho Nacional de Saúde, e suas complementariedades. A pesquisa foi aprovada pelo Comitê de Ética em Pesquisa do Centro de Ciências da Saúde da Universidade Federal da Paraíba, conforme parecer nº 2.243.225/17 e Certificado de apresentação para Apreciação Ética no 71855817.0.0000.5188, sendo conduzida de acordo com os padrões éticos exigidos.

\section{Resultados}

Participaram deste estudo 151 cuidadores, dos quais 118 (78,1\%) eram do sexo feminino, $42(27,8 \%)$ com idades entre 56 e 65 anos, 99 (65,6\%) eram casados ou viviam em união estável, 41 (27,2\%) apresentavam entre cinco e oito anos de estudo, $63(41,7 \%)$ com renda familiar entre $\mathrm{R} \$ 881$ e $\mathrm{R} \$ 1.760$. Sobre os aspectos relacionados ao cuidado dos sobreviventes de acidente vascular encefálico, 62 (41,1\%) eram cônjuges e 58 (38,4\%) filhos desses indivíduos, 116 (76,9\%) cuidavam de pessoas idosas e 135 (89,4\%) moravam na mesma residência, 129 (85,4\%) prestavam cuidados durante todos os dias da semana, 76 (50,3\%) declararam que esses cuidados ocorriam diariamente por 19 horas ou mais e $41(27,2 \%)$ cuidavam daquele indivíduo por um período de tempo de três a cinco anos.

Dentre os principais cuidados prestados, 119 (78,8\%) referiram a medicação; 106 (70,2\%), a higiene corporal; 103 (68,2\%), a locomoção; 103 (68,2\%), o retorno para consultas; $81(53,6 \%)$, os cuidados com a pele; e $75(49,7 \%)$, as eliminações. Dentre os sobreviventes de acidente vascular encefálico, 123 $(81,5 \%)$ apresentavam sequelas relacionadas às alterações motoras, 102 (67,5\%) fraqueza muscular e 57 $(37,7 \%)$ disfagia. Observou-se que a maior parte dos cuidadores apresentou níveis normais de ansiedade $(76,2 \%)$, depressão $(76,8 \%)$ e estresse $(79,5 \%)$ (Tabela 1).

Tabela 1 - Classificação da Escala de Depressão, Ansiedade e Estresse-21. João Pessoa, PB, Brasil, 2017

\begin{tabular}{lccc}
\hline \multirow{2}{*}{ Classificação } & Depressão & Ansiedade & Estresse \\
\cline { 2 - 4 } & $\mathbf{n}(\%)$ & $\mathbf{n}(\%)$ & $\mathbf{n}(\%)$ \\
\hline Normal & $116(76,8)$ & $115(76,2)$ & $120(79,5)$ \\
Leve & $10(6,6)$ & $4(2,6)$ & $10(6,6)$ \\
Moderado & $14(9,3)$ & $19(12,6)$ & $8(5,3)$ \\
Severo & $4(2,6)$ & $2(1,3)$ & $9(6,0)$ \\
Muito severo & $7(4,6)$ & $11(7,3)$ & $4(2,6)$ \\
Total & $151(100,0)$ & $151(100,0)$ & $151(100,0)$ \\
\hline
\end{tabular}

Evidenciou-se que a maioria dos cuidadores não apresentou pensamentos de ideação suicida $(70,9 \%)$. Contudo, $23,2 \%$ dos participantes relataram ter esses pensamentos entre uma ou duas vezes na semana (Tabela 2). 
Tabela 2 - Pensamentos de ideação suicida. João Pessoa, PB, Brasil, 2017

\begin{tabular}{lc}
\hline Classificação (vezes/semana) & n (\%) \\
\hline Nenhum & $107(70,9)$ \\
1 ou 2 & $35(23,2)$ \\
3 ou 4 & $7(4,6)$ \\
$\geq 5$ & $2(1,3)$ \\
Total & $151(100,0)$ \\
\hline
\end{tabular}

A correlação entre a Escala EADS-21 e os pensamentos de ideação suicida apresentaram significância estatística $(\mathrm{p} \leq 0,05)$, sendo observado que à medida que os níveis de ansiedade, depressão e estresse aumentaram, os pensamentos de ideação suicida se elevaramde maneira proporcional (Tabela 3).

Tabela 3 - Correlação entre a depressão, ansiedade e estresse e a os pensamentos de ideação suicida. João Pessoa, PB, Brasil, 2017

\begin{tabular}{lcc}
\hline \multirow{2}{*}{ Correlação } & \multicolumn{2}{c}{ Ideação suicida } \\
\cline { 2 - 3 } & $\mathbf{r}$ & $\mathbf{P}^{*}$ \\
\hline Depressão & 0,395 & $<0,001$ \\
Ansiedade & 0,437 & $<0,001$ \\
Estresse & 0,385 & $<0,001$ \\
\hline${ }^{*}$ Coeficiente Correlação de Spearman
\end{tabular}

\section{Discussão}

As limitações do presente estudo estiveram relacionadas à investigação apenas dos cuidadores formais primários, impedindo a compreensão de maneira mais precisa acerca do impacto do cuidado na vida e saúde desses indivíduos. Além disso, a utilização do método transversal impossibilita a identificação de relações de causa e efeito entre as variáveis analisadas.

Todavia, os dados desta pesquisa têm potencial de contribuir para desenvolvimento e/ou aprimoramento de programas de atenção à saúde de cuidadores, os quais poderiam ser pautados na investigação precoce de processos de adoecimento, na análise da exposição excessiva à sobrecarga de atividades e realização de intervenções para promover maior suporte a esse indivíduo, por meio do apoio familiar e dos profissionais da equipe multidisciplinar.

Dentre os cuidadores investigados, a maioria exibiu valores normais de depressão, ansiedade e estresse, o que poderia estar relacionado ao tempo decorrido desde o episódio de acidente vascular encefálico, uma vez que a maior parte cuida do paciente por período de três a cinco anos, proporcionando maior adaptação à rotina de cuidados. Estudo realizado com 60 familiares de sobreviventes de acidente vascular encefálico em Taiwan evidenciou que as necessidades dos cuidadores em relação ao cuidado prestado diminuíam progressivamente com o tempo de duração da doença, haja vista que durante a hospitalização, esses indivíduos vivenciavam altas cargas de estresse e ansiedade, devido à incerteza do quadro clínico e às desinformações sobre a doença e o respectivo prognóstico ${ }^{(3)}$.

Associado a isso, a vivência de sentimentos positivos relacionados ao cuidado poderia favorecer a redução dos níveis de ansiedade, estresse e depressão, destacando-se a afetividade pelo familiar, os momentos felizes de interação, a solidariedade, a gratidão, a valorização, o comprometimento, o bem-estar e a percepção da importância das atividades desempenhadas para vida do outro ${ }^{(6)}$.

Em contrapartida, o cuidado de indivíduo dependente está relacionado à presença de sobrecarga e de adoecimento físico e psíquico, principalmente na ausência de apoio familiar, em que todas as responsabilidades recaem sobre um único indivíduo, que tenta equilibrar a prestação de cuidados diários ao familiar dependente, com todas as demais obrigações referentes à própria vida ${ }^{(4,11)}$.

Nesse sentido, percebe-se a vivência de conflitos relacionados a esse processo de cuidado. Embora, em muitas ocasiões, existam momentos de prazer que podem ressignificar as atividades desenvolvidas, são frequentes os episódios de sofrimento durante a prestação do cuidado, os quais estão mais associados aos problemas de sobrecarga de tarefas realizadas por um 
único indivíduo, do que especificamente ao desempenho de tais atividades, repercutindo diretamente na ausência de apoio familiar como ponto crucial no adoecimento desses cuidadores.

Pesquisas revelaram a influência negativa do cuidado para vida do cuidador, interferindo na rotina diária, na renda individual e familiar, no trabalho e/ou estudos, no lazer e nos relacionamentos afetivos ${ }^{(1,5-6,12)}$. Os prejuízos decorrentes desse cuidado prolongado não se restringem apenas à esfera individual do cuidador, podendo interferir na qualidade das ações executadas e na própria integridade física do paciente, haja vista que a sobrecarga representa importante fator de risco para violência e negligência ${ }^{(6)}$.

Diante disso, a utilização de estratégias de enfrentamento poderiam ser ferramentas relevantes para redução da sobrecarga e do estresse nesses cuidadores, como a busca por qualificação, o apoio social de familiares, colegas, amigos e profissionais de saúde, o fortalecimento da fé, a resolução dos problemas enfrentados e a organização hábil do tempo, de modo a destinar momentos para o autocuidado e o $\operatorname{lazer}^{(8)}$. Além disso, faz-se necessária a promoção do autocuidado nesses indivíduos, uma vez que muitos priorizam a prestação de cuidados ao familiar dependente em detrimento do cuidado com a própria saúde, provocando o adoecimento ${ }^{(6,13)}$.

Em relação à ideação suicida, observou-se que pequena parcela dos cuidadores apresentou tais pensamentos entre uma ou duas vezes na semana, o que representa sinal de alerta, haja vista que o cuidado diário ao familiar dependente pode potencializar a vivência de sofrimento e aumentar a frequência de pensamentos relacionados à morte. Corroborando com esses achados, estudo realizado na China identificou que o cuidado ao familiar sobrevivente de acidente vascular encefálico provocou o desenvolvimento de sentimentos negativos, os quais eram intensificados progressivamente, à medida que o tempo de cuidado se elevava ${ }^{(14)}$.

A ideação suicida está frequentemente atrelada à presença constante de vivências de sofrimento e alterações psicológicas, sobretudo, a depressão, tornando o indivíduo mais vulnerável ao sofrimento emocional à ocorrência de pensamentos de frustração e inutilidade ${ }^{(4,14-15)}$. A depressão é um problema bastante comum entre cuidadores de pessoas que requerem cuidados de longa duração, como os sobreviventes de acidente vascular encefálico, tornando-se fator que pode predispor o cuidador à violência autoinflingida e ao suicídio( ${ }^{(7,16)}$.

As próprias sequelas decorrentes do acidente vascular encefálico, também, podem desencadear sofrimento no cuidador, gerando maior risco de desenvolvimento de depressão e pensamentos de ideação suicida. Outro estudo realizado com 136 cuidadores de pessoas com sequelas de acidente vascular encefálico identificou o comprometimento da qualidade de vida geral e de todos os domínios, sendo mais elevado o sofrimento mental nos cuidadores de indivíduos com maior dependência de cuidado, sendo a dimensão psicológica a primeira a ser $\operatorname{afetada}^{(17)}$.

Diante desse aspecto, ressaltar destaca-se a questão de gênero que permeia o processo de indicação do familiar para realização dos cuidados e de como isso afeta as dimensões da vida desse indivíduo. 0 papel da mulher no cuidado aos doentes é algo histórico e que permanece nos dias atuais, refletindo diretamente na escolha da esposa, filha ou neta como a principal cuidadora, a qual também realiza os afazeres da casa e o cuidado do restante da família, vivenciando diariamente intensa sobrecarga e acúmulo de tarefas, o que frequentemente resulta no adoecimento físico e psíquico.

Mediante a correlação, foi possível identificar que os pensamentos de ideação suicida se elevavam, à medida que os níveis de depressão, ansiedade e estresse aumentavam. Este achado poderia ser justificado pela complexidade que envolve o cuidado a uma pessoa com sequela de acidente vascular encefálico, sobretudo, membro familiar, uma vez que é um evento inesperado e que gera incapacidades temporárias e/ 
ou permanentes, provocando alterações na organização familiar, nas relações de afeto e na estrutura financeira da casa ${ }^{(4)}$.

Diante disso, muitos cuidadores vivenciam momentos de tensão, ansiedade, estresse e depressão, principalmente por terem que assumir novo papel para o qual não apresentam conhecimentos ou mesmo capacitação, o que pode desencadear fortes emoções, sentimentos de incapacidade e de revolta e ausência de perspectivas futuras, proporcionando maior vulnerabilidade ao adoecimento psíquico e ao suicídio $^{(7,18-19)}$.

A invisibilidade do cuidador também representa problemática relevante no Brasil e em âmbito internacional, a qual pode contribuir para o sofrimento desse indivíduo, haja vista que a carência de suporte por parte dos familiares e profissionais de saúde provoca a negligência do estado de saúde e das próprias necessidades individuais desses sujeitos, o que favorece o aumento de sintomas depressivos, que se não tratados adequadamente, podem resultar na tentativa de suicídio ${ }^{(4,13,20)}$.

Nesse sentido, faz-se necessário que o profissional de saúde, durante a assistência a pacientes com sequelas de acidente vascular encefálico, inclua o cuidador como parte essencial do plano de cuidados, buscando esclarecer dúvidas, orientar sobre as técnicas para o cuidado, investigar precocemente a presença de processos de sobrecarga e adoecimento, ofertar apoio necessário e promover a valorização das funções desempenhadas por esse indivíduo ${ }^{(6,17)}$.

\section{Conclusão}

Observou-se que a maioria dos cuidadores apresentou níveis normais de depressão, ansiedade e estresse, com ausência de pensamentos de ideação suicida. A correlação entre as variáveis evidenciou que níveis elevados de depressão, ansiedade e estresse favoreceram o aumento da frequência de pensamentos de ideação suicida entre os cuidadores, o que sinaliza a necessidade de se investigar precocemente o desen- volvimento de processos de desgaste que podem resultar em graves prejuízos para saúde e qualidade de vida desses indivíduos.

\section{Colaborações}

Costa TF, Bezerra TA, Pimenta CJL, Silva CRR, Ferreira GRS e Costa KNFM contribuíram na concepção e projeto ou análise e interpretação dos dados, redação do artigo, revisão crítica relevante do conteúdo intelectual e aprovação final da versão a ser publicada.

\section{Referências}

1. Zhang H, Lee DT. Meaning in stroke family caregiving: A literature review. Geriatr Nurs. 2017; 38(1):48-56. doi: https://doi.org/10.1016/j.gerinurse.2016.07.005

2. Hu P, Yang MMQ, Kong L, Hu L, Zeng BSL. Relationship between the anxiety/depression and care burden of the major caregiver of stroke patients. Medicine (Baltimore). 2018; 97(4):e12638. doi: http://dx.doi.org/10.1097/ MD.0000000000012638

3. Tsai PC, Yip PK, Tai JJ, Lou MF. Needs of family caregivers of stroke patients: a longitudinal study of caregivers' perspectives. Patient Prefer Adherence. 2015; 9:449-57. doi: http://dx.doi. org/10.2147/PPA.S77713

4. Pesantes MA, Brandt LR, Ipince A, Miranda JJ, Diez-Canseco F. An exploration into caring for a stroke-survivor in Lima, Peru: emotional impact, stress factors, coping mechanisms and unmet needs of informal caregivers. eNeurological Sci. 2017; 6:33-50. doi: http://dx.doi.org/10.1016/j. ensci.2016.11.004

5. Menon B, Salini P, Habeeba K, Conjeevaram J, Munisusmitha K. Female caregivers and stroke severity determines caregiver stress in stroke patients. Ann Indian Acad Neurol. 2017; 20(4):418-24. doi: http://dx.doi.org/10.4103/aian.AIAN_203_17

6. Couto AM, Castro EAB, Caldas CP. Experiences to be a family caregiver of dependent elderly in the environment. Rev Rene. 2016; 17(1):7685. doi: http://dx.doi.org/10.15253/21756783.2016000100011 
7. Ifeanyi UC, Sa'ad FS, Martin OC, Monday MS, Onyinyechukwu NJ. Prevalence of depression among primary caregivers of stroke survivors in Nigeria. Middle East J Rehabil Health. 2018; 5(2):e65080. doi: http://dx.doi.org/10.5812/ mejrh.65080

8. Kumar R, Kaur S, Reddemma K. Burden and coping strategies in caregivers of stroke survivors. J Neurol Neurosc. 2015; 6(1):1-5. doi: http:// dx.doi.org/10.21767 / 2171-6625.s10005

9. Vignola RC, Tucci AM. Adaptation and validation of the depression, anxiety and stress scale (DASS) to Brazilian Portuguese. J Affect Disord. 2014; 155:104-9. doi: http://dx.doi.org/10.1016/j. jad.2013.10.031

10. Cubas MA, Nóbrega MML. Atenção Primária em Saúde: Diagnósticos, Resultados e Intervenções de Enfermagem. Rio de Janeiro: Elsevier; 2015.

11. Fogaça NJ, Carvalho MM, Montefusco SRA. Perceptions and feeling of the Family member/caregivers expressed before the patient on home care. Rev Rene. 2015; 16(6):848-55. doi: http://dx.doi. org/10.15253/2175-6783.2015000600011

12. Tsai YH, Lou MF, Chu TL, Chen YJ, Liu HE. Mediating effects of burden on quality of life for caregivers of first-time stroke patients discharged from the hospital within one year. BMC Neurol. 2018; 18:50. doi: http://dx.doi.org/10.1186/s12883018-1057-9

13. Nascimento MGG, Martins PCF, Resck ZMR, Dázio EMR, Terra FS. Self-care to elderly after cerebrovascular accident: caregiver and academics experiences. Rev Rene. 2015; 16(5):682-9. doi: http://dx.doi.org/10.15253/2175-6783.2015000500009
14. Guo Y, Liu Y. Family functioning and depression in primary caregivers of stroke patients in China. Int J Nurs Scienc. 2015; 2(2):184-9. doi: https://doi. org/10.1016/j.ijnss.2015.05.002

15. Chamberlain L, Anderson C, Knifton C, Madden G. Suicide risk in informal carers of people living with dementia. Nurs Older People. 2018; 30(5):20-5. doi: http://dx.doi.org/10.7748/nop.2018.e1035

16. Gómez MAH, Domínguez MJF, Ramos MAB, Pérez MTA, Domínguez MJA, Ramos AIS, et al. Depression and burden in the caretaking of elderly. Rev Esp Salud Publica [Internet]. 2019 [cited Sep 11, 2019]; 93:e1-e10. Available from: https:// www.mscbs.gob.es/biblioPublic/publicaciones/ recursos_propios/resp/revista_cdrom/VOL93/O_ BREVES/RS93C_201908038.pdf

17. Costa TF, Gomes TM, Viana LRC, Martins KP, Costa KNFM. Stroke: patient characteristics and quality of life of caregivers. Rev Bras Enferm. 2016; 69(5):933-9. doi: http://dx.doi. org/10.1590/0034-7167-2015-0064

18. Zhu W, Jiang Y. Determinants of caregiver burden of patients with haemorrhagic stroke in China. Int J Nurs Pract. 2019; 25(2):e12719. doi: http:// dx.doi.org/10.1111/ijn.12719

19. Kwon J, Park EC, Kim W, Choi DW, Jang SI. Depressive symptoms in individuals with family members requiring ADL assistance. Environ Health Prev Med. 2019; 24(1):49. doi: http://dx.doi. org/10.1186/s12199-019-0804-x

20. Ninomiya S, Tabuchi K, Rahman MM, Kobayashi T. Factors associated with mental health status among older primary caregivers in Japan. Inquiry. 2019; 56:46958019859810. doi: http://dx.doi. org/10.1177/0046958019859810

\section{(cc) BY}

Este é um artigo de acesso aberto distribuído sob os termos da Licença Creative Commons 